\title{
Prevalence of Extended Spectrum B-Lactamase (ESBL) Producing Bacteria among the Clinical Samples in and around a Tertiary Care Centre in Nerul, Navi Mumbai, India
}

\author{
Betsy Andrews, Shrikrishna Joshi*, Rita Swaminathan, \\ Jyoti Sonawane and Keertana Shetty
}

D Y Patil Medical College and Hospital, Nerul, India

*Corresponding author

\begin{tabular}{|l|}
\hline Ke y w o r d s \\
Antibiotic abuse, \\
$\begin{array}{l}\text { Extended spectrum } \\
\beta \text {-lactamase }\end{array}$ \\
\hline Article Info \\
\hline $\begin{array}{l}\text { Accepted: } \\
\text { 26 February } 2018 \\
\text { Available Online: } \\
\text { 10 March } 2018\end{array}$ \\
\hline
\end{tabular}

\section{Introduction}

Extended-spectrum beta-lactamases (ESBL) are enzymes that confer resistance to betalactam antibiotics, including penicillins, cephalosporins, and the monobactam aztreonam. Infections with ESBL-producing organisms have been associated with poor clinical outcomes. Community and hospitalacquired ESBL-producing Enterobacteriaceae are prevalent worldwide ${ }^{[1]}$. Reliable
To study the prevalence of extended spectrum $\beta$-lactamase (ESBL) producing bacteria and its sensitivity pattern to commonly used antibiotics in a tertiary care centre in Navi Mumbai. The study was conducted from June 2016 to July 2017 in Microbiology Department of our tertiary health care centre. A total of 2850 sample was studied, out of which 812 (54.79\%) ESBL producing bacteria was detected by using Clinical Laboratory Standard Institute (CLSI) guidelines that described the phenotypic confirmatory test along with routine antibiotic susceptibility testing. ESBL production was confirmed in 812 (54.79\%) isolates. The isolates of E. coli $(45.50 \%)$ were the most common ESBL producers. Maximum ESBL isolates were obtained from urine samples (44.3\%) and male patients $(56.4 \%)$. Surgical ward showed highest prevalence $(21.1 \%)$ and age group between 51 and 60 were mostly affected (19.9\%). This study conducted in D Y Patil Hospital, Nerul, Navi Mumbai shows high prevalence of ESBL production among Gram negative bacteria. E.coli showed highest prevalence i.e. 45.5\%. Colistin showed $100 \%$ sensitivity followed by Imipenem which showed $98.2 \%$. Prevalence of ESBL producers was more prevalent in urine sample among males than females. Timely administration of sensitive antibiotic and avoiding antibiotic abuse will help to lessen the burden of ESBL 
enzyme was discovered and named TEM-2. It was identical in biochemical properties to the more common TEM-1 but differed by a single amino acid with a resulting change in the isoelectric point of the enzyme. These two enzymes are the most common plasmidmediated beta-lactamases in gram-negative bacteria, including Enterobacteriaceae, Pseudomonas aeruginosa, Haemophilus influenzae, and Neisseria gonorrhoeae. TEM1 and TEM-2 hydrolyze penicillins and narrow spectrum cephalosporins, such as cephalothin or cefazolin. However, they are not effective against higher generation cephalosporins with an oxyimino side chain, such as cefotaxime, ceftazidime, ceftriaxone, or cefepime. Consequently, when these antibiotics were first introduced, they were effective against a broad group of otherwise resistant bacteria. [3-5] Bacteria producing ESBL are spread mostly through hospital staff like doctors, nurses or other healthcare professionals. They are vastly responsible for causing infections such as UTI, diarrhoea, skin infection and pneumonia. Symptoms of an ESBL infection depends on the site of bacterial colonization, such as burning micturation in case of UTI, loss of appetite and presence of blood in stool in case of GIT infection and rashes in case of skin infection. Since these bacteria are highly resistant, antibiotics should be administered only after performing an antibiotic sensitivity testing according to CLSI guidelines.

\section{Materials and Methods}

The study was conducted in microbiology department at D. Y Patil Medical College and Hospital. The hospital is an 800 bedded multispeciality hospital with latest advances, catering to more than 1000 patients in a day. All the clinical samples collected in a sterile container for bacteriological study were included. For isolation and identification of the bacteria, following media were used:
Blood agar, Chocolate agar, MacConky agar along with series of biochemical test like Indole, Citrate, Urease and TSI. The bacterial isolates were then tested for antimicrobial susceptibility test by the disc diffusion method according to the guidelines of Clinical and Laboratory Standard Institute (CLSI) ${ }^{\text {[6]. The }}$ following antibiotics discs were used in the sensitivity test: Imipenem $(10 \mathrm{mcg})$, Colistin (10 mcg), Amikacin (10 mcg),Gentamicin (10 mcg), Cefotaxime (30 mcg), Ceftrioxone (30 mcg), Ceftazidime (30 mcg), Cefepime (30 $\mathrm{mcg})$, Ciprofloxacin(5 mcg), Nitrofurantoin (100 mcg), Cotrimoxazole (25 $\mu \mathrm{g})$, Tobramycin $(10 \mathrm{mcg})$, Norfloxacin $(10 \mathrm{mcg})$, PipracillinTazobactum (100/10mcg), and Ampicillin (10mcg).

\section{Testing for the ESBL production}

The ESBLs detection was carried out by modified double disc synergy test using Cefepime along with the third generation Cephalosporins ${ }^{[7] .}$ All the strains which will show a diameter of less than $27 \mathrm{~mm}$ for Cefotaxime and less than $25 \mathrm{~mm}$ for Ceftriaxone were selected for checking the ESBLs production. The ESBL production was tested by the Modified Double Disc Synergy Test by using a disc of Amoxicillinclavulanate $(20 / 10 \mu \mathrm{g})$ along with four Cephalosporins (Cefotaxime, Ceftriaxone, Cefpodoxime and Cefepime). A lawn culture of the organisms was made on a MuellerHinton agar plate, as recommend by CLSI ${ }^{[8]}$. A disc which contained Amoxicillinclavulanate $(20 / 10 \mu \mathrm{g})$ was placed in the centre of the plate. The discs of third generation cephalosporin and fourth generation cephalosporin was placed $15 \mathrm{~mm}$ and $20 \mathrm{~mm}$ apart respectively, centre to centre to that of the Amoxicillin-clavulanate disc ${ }^{[9]}$. Any distortion or increase in the zone towards the disc of Amoxicillin-clavulanate was considered as positive for the ESBLs production. 


\section{Results and Discussion}

A total of 2850 samples were obtained out of which 1482 gave positive bacterial growth (Fig. 1). Prevalence of ESBL producers were estimated to be $54.79 \%$ i.e. 812 out of the total 1482 were ESBL producing bacteria (Fig. 2).

Prevalence was seen more among the male patients than female patients. $56.4 \%$ of the males showed to be infected with ESBL whereas female ratio was estimated to be 43.5 $\%$ (Fig. 3). The age group of 51-60 showed maximum infection with EBSL (19.9\%) followed by age group of 21-30 (19.7\%). The least affected age group was 11-20 (0.05\%) (Fig. 4). Patients admitted to surgical ward was mostly affected $(21.10 \%)$ followed by MICU (20.5\%) (Fig. 5).
Maximum number of ESBL producers were isolated from urine sample (44.30\%) followed by pus (32.2\%) (Fig. 6). Among the different gram negative bacteria obtained and studied, E. coli showed the maximum number of ESBL production i.e. $45.5 \%$ (Fig. 7).

All the ESBL producing organisms were studied for their antibiotic sensitivity pattern and organisms showed $100 \%$ sensitivity to Colistin and $98.2 \%$ sensitivity to Imipenem. The sensitivity percentage to the antibiotics is listed in the table 1. Other antibiotics including Ceftazidime, Cefepime, Nitrofurantoin and Ampicillin showed complete resistance along with Cefotaxime and Ceftriaxone.

Fig.1 Shows total bacterial growth obtained in the study

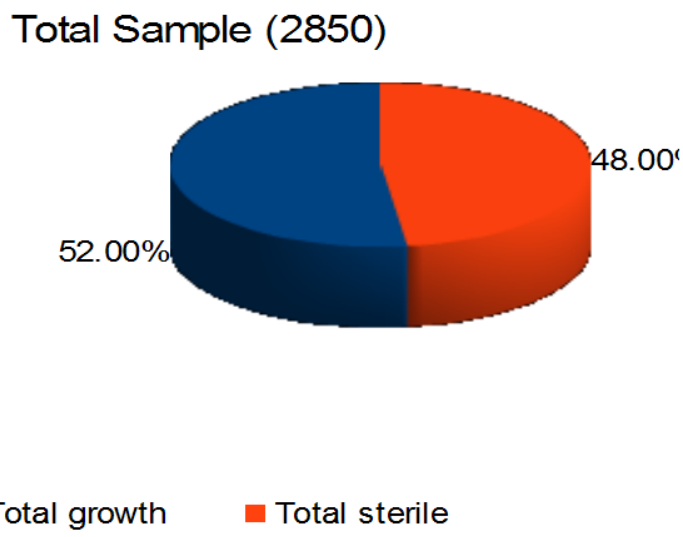

Fig.2 Shows total number of ESBL producers obtained

Total ESBL producer (812 i.e 54.79\%)

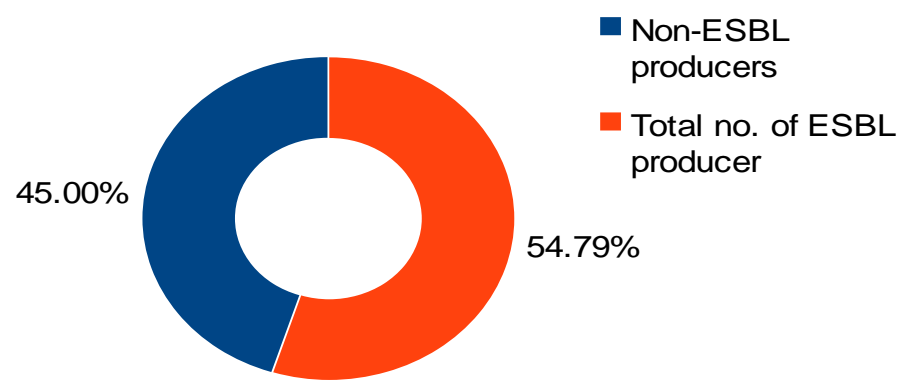


Fig.3 Shows the prevalence of ESBL among male and female patients

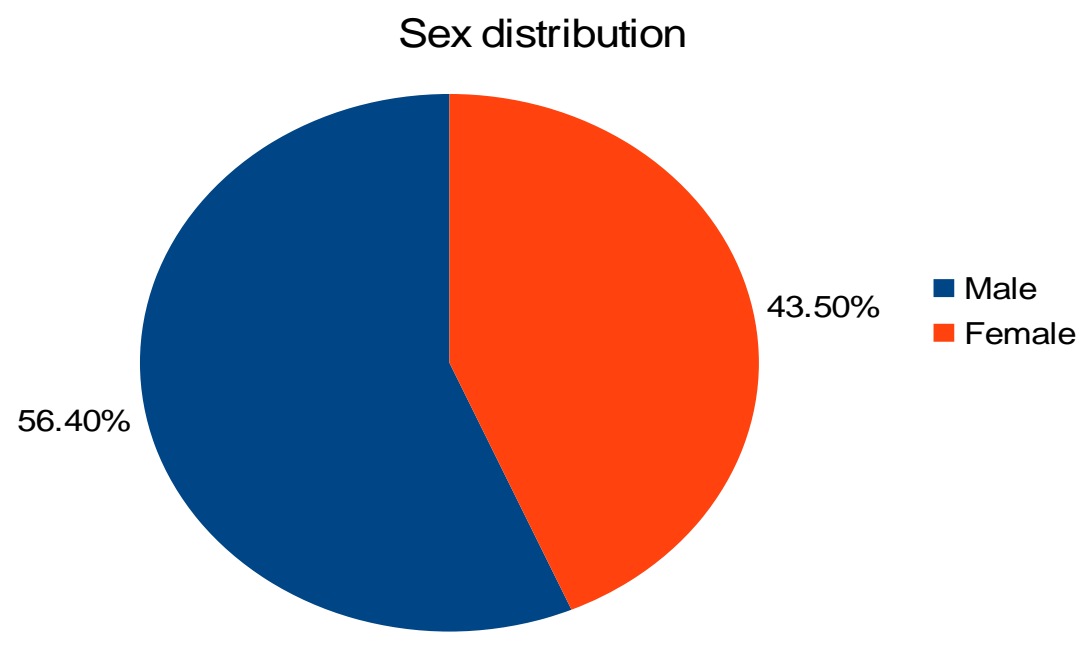

Fig.4 Shows age wise distribution of ESBL. 1 - 10: 8.30\%, 11- 20: 0.05\%, 21-30: 19.7\%, 31-40: 14\%, 41-50: 13.7\%, 51-60: 19.9\%, 60-70: 12\%, 71-80 and above: 16.4\%

\section{Agewise Distribution}

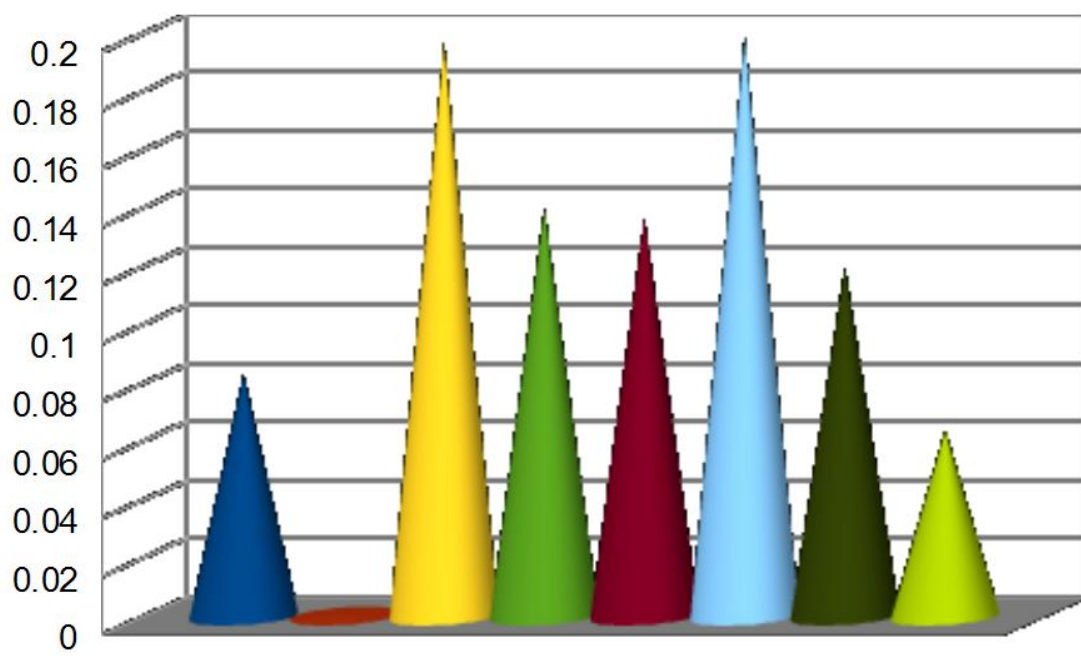

$1-10$

ㅁ $11-20$

$21-30$

- $31-40$

- $41-50$

$51-60$

- $61-70$

71 - 80 and above. 
Fig.5 Shows ward wise distribution among ESBL. Outpatient department: 14.9\%, Surgical ward: 21.1\%, OBGY: 5.9\%, Peads: 6.1\%, MICU: 20.5\%, Medical ward: 16.5\%, Urology: 1.4\%, Ortho: 4.9\%, PICU: 0.61\%, Medicine: 6.2\%, Nephrology: 0.49\%, Ophthalmology: 0.49\%, Skin: 0.24\%

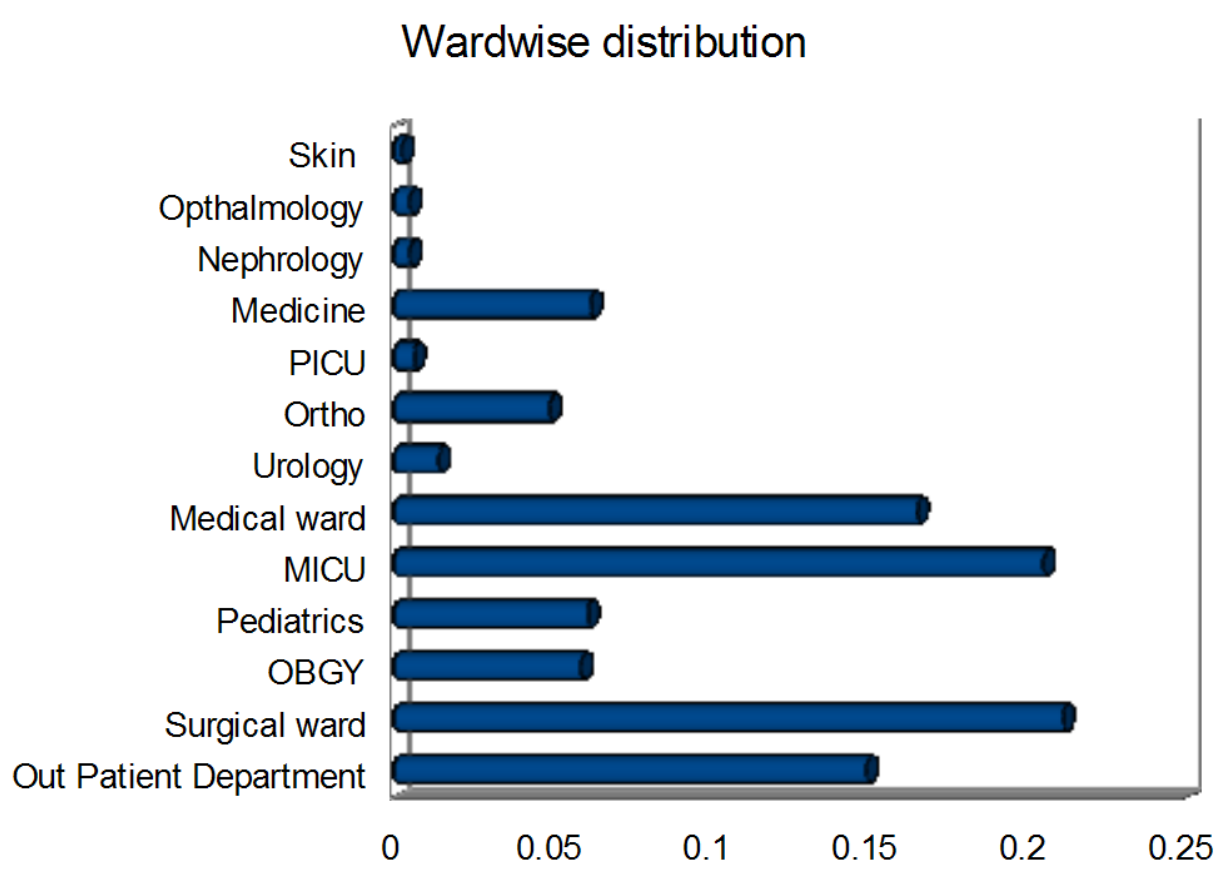

Fig.6 Shows the growth of ESBL producers among the different samples. Sputum: 4.1\%, Pus: $32.2 \%$, Urine: $44.3 \%$, Tips: $11.8 \%$, Blood and tissue: $0.73 \%$, Other body fluids: $4.43 \%$, Stool:

\section{Samplewise distribution}

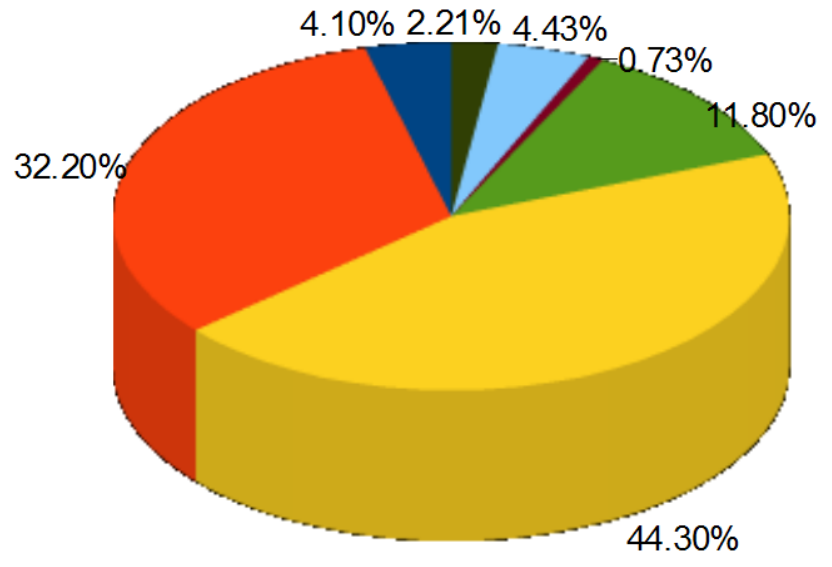

- Sputum

Pus

Urine

- Tips

Blood and tissues

Other body fluids

- Stool 
Fig.7 Shows the ESBL production among the different gram negative bacteria obtained in the study. E. coli: $45.5 \%$, Klebsiella pneumoniae: $24.1 \%$, Klebsiella oxytoca: $9.6 \%$, Pseudomonas species: $2.9 \%$, Pseudomonas aeruginosa: $4.4 \%$, Proteus vulgaris: $2.2 \%$, Proteus mirabilis:

0.98\%, Citrobacter freundii: $2.9 \%$, Citrobacter koseri: $1.2 \%$, Acinetobacter species:

5.1\% and Enterobacter species: $0.9 \%$

\section{ESBL Producers}

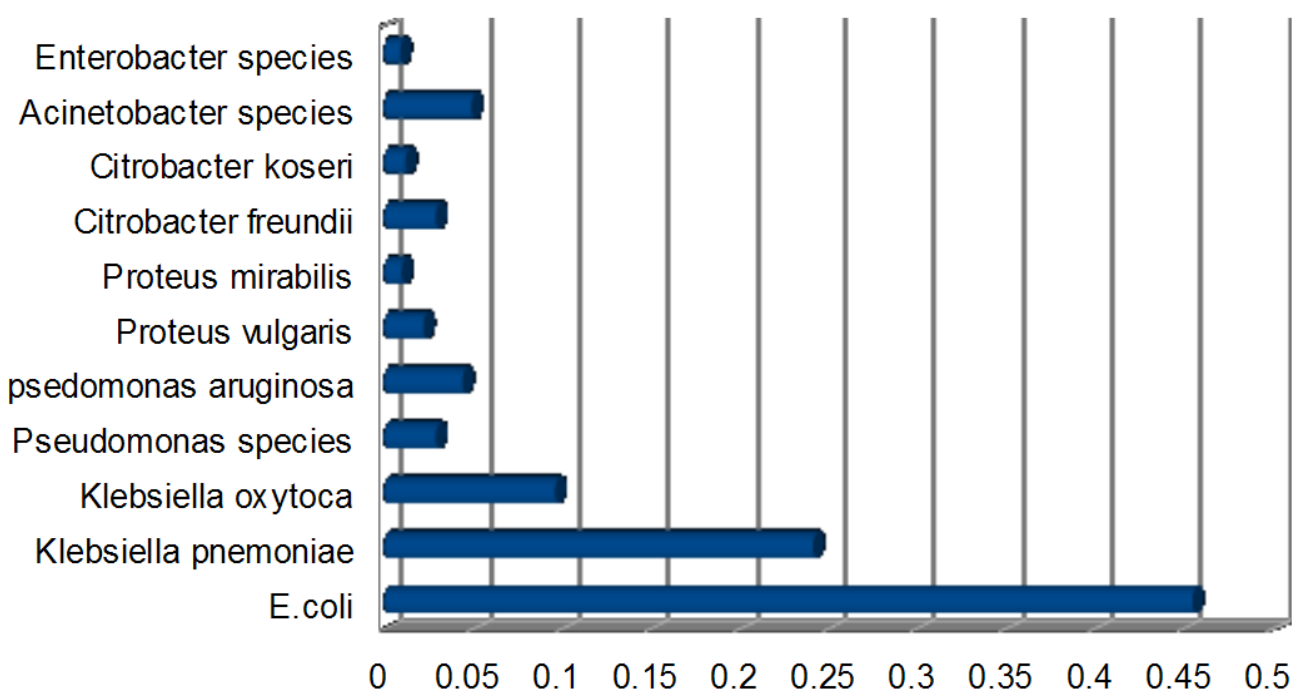

Table.1 The sensitivity percentage to antibiotics

\begin{tabular}{|c|c|c|}
\hline SR. & Name of the antibiotic & Sensitivity percentage \\
\hline 1 & Colistin & $100.00 \%$ \\
\hline 2 & Imipenem & $98.20 \%$ \\
\hline 3 & Amikacin & $94.90 \%$ \\
\hline 4 & Gentamycin & $93.34 \%$ \\
\hline 6 & Ciprofloxacin & $74.20 \%$ \\
\hline 7 & Co-trimoxazole & $73.80 \%$ \\
\hline 8 & Tobramycin & $72.00 \%$ \\
\hline 9 & Piperacillin-Tazobactum & $70.50 \%$ \\
\hline 10 & Norfloxacin & $50.20 \%$ \\
\hline
\end{tabular}

Moland and colleagues have shown that ESBL-producing isolates were found in $75 \%$ of 24 medical centers in the United States (23 Moland et al., 2002) ${ }^{[10]}$ while in other studies around USA, $4.244 \%$ of gram negative bacteria were found to be ESBL producers (24-26 Saurian s et al., 2000; Mathai et al., 2001; Winokur et al., 2001) ${ }^{[11-13]}$. Spain has seen a prevalence of $20.8 \%$ (27 Romero et al., 2007) ${ }^{[14]}$, Taiwan $28.4 \%$ (28 Kuo et al., 2007) ${ }^{[15]}$, Turkey $78.6 \%$ (29 Hos, oglu et al., 2007) ${ }^{[16]}$, Algeria 20\% (30 Messai et al., 2008) ${ }^{[17]}$ and China 51\% (31 Xiong et al., $2002)^{[18]}$. The studies conducted in India also show high prevalence of ESBL producers. (59.9\%, Telangana, Hema bindu et al., 2015) 
[19] In our study, we have also reported a prevalence of $54.97 \%$ of ESBLs which is of a very high concern. E. coli has shown a prevalence of $45.5 \%$, majority of it being in the urine samples. This could be because of the injudicious use of drugs for urinary tract infections.

This study along with the other studies concludes that prevalence of ESBL producing bacteria is rising in an alarming way. The resistance to the antibiotic is ever increasing among the bacteria due to antibiotic abuse and hence timely reporting of ESBL producing organisms and administration of sensitive antibiotic along with proper awareness is need of the hour.

The study was aimed to understand the prevalence of ESBL producing Gram Negative Bacteria among the microbial samples received in the microbiology department of D.Y Patil Medical College and Hospital in Nerul, Navi Mumbai. The data shows $54.97 \%(812 / 1482)$ prevalence of ESBL producers among the positive samples. The prevalence among the sexes was $56.4 \%$ and $46.5 \%$, more common in men than in women respectively. The infection rate was $19.9 \%$ among the patients belonging to the age group of 51-60. Surgical ward showed highest prevalence i.e. $21.1 \%$ of ESBL producers. Maximum number of ESBL producing bacteria i.e. $44.3 \%$ was isolated from urine sample and $E$. coli was identified to be the highest producer of beta- lactamase enzyme which is $45.5 \%$. Colistin showed $100 \%$ sensitivity to all the ESBL producing bacteria.

\section{References}

Ben-Ami R, Rodríguez-Baño J, Arslan H, et al., A multinational survey of risk factors for infection with extendedspectrum beta-lactamase-producing enterobacteriaceae in nonhospitalized patients. Clin Infect Dis 2009; 49:682.

Bradford PA. Extended-spectrum betalactamases in the 21st century: characterization, epidemiology, and detection of this important resistance threat. Clin Microbiol Rev 2001; 14:933.

Doi Y, Park YS, Rivera JI, et al., Communityassociated extended-spectrum $\beta$ lactamase-producing Escherichia coli infection in the United States. Clin Infect Dis 2013; 56:641.

Hima Bindu et al., Prevalence of ESBL Production in Escherichia coli and Klebsiella spp from Different Clinical Samples A Study in a Teaching Hospital in Telangana, India. Int. J. Curr. Microbiol. App. Sci (2015) 4(3): 236243

Hos soglu, S., Gundes, S., Kolayli, F., et al., 2007. Extended-spectrum beta lactamases in ceftazidime-resistant Escherichia coli and Klebsiella pneumoniae isolates in Turkish hospitals. Indian J. Med. Microbiol., 25(4): 346350.

Kaur J, Chopra S, Sheevani, Mahajan G (2013) Modified Double Disc Synergy Test to Detect ESBL Production in Urinary Isolates of Escherichia coli and Klebsiella pneumonia. J ClinDiagn Res 7: 229-233.

Kliebe C, Nies BA, Meyer JF, et al., Evolution of plasmid-coded resistance to broad-spectrum cephalosporins. Antimicrob Agents Chemother 1985; 28:302.

Kuo, K.C., Shen, Y.H., Hwang, K.P. 2007.Clinical implications and risk factors of extended-spectrum betalactamase producing Klebsiella pneumoniae infection in children: a casecontrol retrospective study in a medical center in southern Taiwan. $J$. Microbiol. Immunol. Infect., 40(3):48 
254.

Mathai, D., Lewis, M.T., Kugler, K.C.,Pfaller, M.A., Jones, R.N. 2001.Antibacterial activity of 41 antimicrobials tested against over 2773 bacterial isolates from hospitalized patients with pneumonia: I-results from the SENTRY antimicrobial surveillance program (North America, 1998).Diag. Microbiol. Infect. Dis., 39(2):105 116.

Messai, Y., Iabadene, H., Benhassine, T., etal. 2008. Prevalence and characterization of extendedspectrum-lactamases in Klebsiella pneumoniae in Algiers hospitals (Algeria). Pathologie Biologie, 56(5): 319325.

Moland, E.S., Black, J.A., Ourada, J., Reisbig, M.D., Hanson, N.D., Thomson, K.S. 2002. Occurrence of newer betalactamases in Klebsiella pneumoniae isolates from 24 U.S. Hospitals. Antimicrob. Agents Chemother., 46(12): 383742.

Paterson DL, Bonomo RA. Extendedspectrum beta-lactamases: a clinical update. Clin Microbiol Rev 2005; 18:657.

Romero, E.D.V., Padilla, T.P., Hernandez, A.H., et al., 2007. Prevalence of clinical isolates of Escherichia coli and Klebsiella spp. Producing multiple extended-spectrum -lactamases. Diag. Microbiol. Infect. Dis., 59(4): 433437.

Saurian S, J.M., Quale, V.M., Manikal, E.,
Oydna, Landman, D. Antimicrobial resistance in Enterobacteriaceae in Brooklyn, NY: epidemiology and relation to antibiotic usage patterns. $J$. Antimicrob. Chemother., 45(6):895 898.

Schmiedel J, Falgenhauer L, Domann E, Bauerfeind R, Prenger-Berninghoff E, et al., (2014) Multiresistant extendedspectrum $\quad \beta$-lactamaseproducing Enterobacteriaceae from humans, companion animals and horses in central Hesse, Germany. BMC Microbiology 14:1-13.

Tzelepi E, Gaikkoupi P, Sofianou D, Loukova $\mathrm{V}$, Kemeroglou A, et al., (2000) JClinMicrobiol 38:542-546.

Wayne PA (2009) Performance standards for antimicrobial susceptibility testing; nineteenth informational supplement M100-S19. Clinical and Laboratory Standards Institute.

Winokur, P.L., Canton, R., Casellas, J.M., Legakis, N. 2001. Variations in the prevalence of strains expressing an extended spectrum -lactamase phenotype and characterization of isolates from Europe, the Americas, and the Western Pacific region. Clin. Infect. Dis., 32(Suppl. 10): S94S103.

Xiong, Z., Zhu, D., Zhang, Y., Wang, F.2002. Extended-spectrum betalactamase in Klebsiella pneumoniae and Escherichia coli isolates. Zhonghua Yi Xue Za Zhi, 82(21):1476 1479.

\section{How to cite this article:}

Betsy Andrews, Shrikrishna Joshi, Rita Swaminathan, Jyoti Sonawane and Keertana Shetty. 2018. Prevalence of Extended Spectrum B-Lactamase (ESBL) Producing Bacteria among the Clinical Samples in and around a Tertiary Care Centre in Nerul, Navi Mumbai, India. Int.J.Curr.Microbiol.App.Sci. 7(03): 3402-3409. doi: https://doi.org/10.20546/ijcmas.2018.703.392 